\title{
Rotações Aleatórias
}

\section{Random Rotations}

\author{
Lucas H. Oliveira ${ }^{1}$, Marcel Novaes ${ }^{* 1}$ \\ ${ }^{1}$ Universidade Federal de Uberlândia, Instituto de Física, Uberlândia, MG, Brasil.
}

\begin{abstract}
Recebido em 16 de julho de 2020. Revisado em 23 de setembro de 2020. Aceito em 22 de outubro de 2020
As rotações estão entre as mais simples e mais importantes das operações lineares, e o grupo das rotações é uma estrutura fundamental tanto para a física quanto para a geometria. Neste artigo, apresentamos uma discussão introdutória sobre rotações aleatórias, o que pode ser visto como um exemplo da teoria mais geral dos operadores aleatórios ou das matrizes aleatórias, área que atualmente encontra inúmeras aplicações. Discutimos a estrutura do grupo ortogonal, um algoritmo para gerar rotações aleatórias e resultados para as distribuições de probabilidade dos elementos de matriz e dos autovalores.
\end{abstract}

Palavras-chave: Rotações, Aleatoriedade, Probabilidade, Grupo ortogonal.

Rotations are among the simplest and most important of linear operations, and the group of rotations is a fundamental structure both for physics and geometry. In the article, we present an introductory discussion about random rotations, which can be seen as an example from the more general theory of random operators or random matrices, area which finds countless applications. We discuss the structure of the orthogonal group. an algorithm for generating random rotations and results for the probability distributions of matrix elements and eigenvalues. Keywords: Rotations, Randomness, Probability, Orthogonal group.

\section{Introdução}

No começo de um jogo de futebol, o juiz coloca a bola no centro do campo e, depois do apito, o primeiro chute a faz rolar. Ao cabo de aproximadamente 45 minutos, o primeiro tempo acaba e vem o intervalo. Quando o segundo tempo vai começar, o juiz coloca novamente a bola no centro do campo. Muito bem, eis que temos um teorema: Quando a bola é recolocada para o início do segundo tempo, existem dois pontos sobre a sua superfície que voltam exatamente à mesma posição que tinham antes do início do jogo.

Como podemos ter certeza disso? Afinal, não sabemos o que ocorreu durante a partida e o juiz pode posicionar a bola de maneira imprevisível (ele pode posicioná-la de maneira arbitrária, afinal ele é o árbitro).

Acontece que, seja lá como a bola for colocada, se a posição do centro no início do segundo tempo coincide com a posição do centro no início do primeiro, então a única alteração que pode ter ocorrido em sua configuração é uma rotação: a segunda posição pode ser obtida rodando a primeira de alguma forma (tal fato é conhecido como Teorema da Rotação, de Euler). Ora, toda rotação no espaço tridimensional é especificada por um eixo em torno do qual é realizada e por um ângulo, e qualquer rotação aplicada à superfície de uma esfera sempre deixa dois pontos fixos: aqueles que pertencem ao eixo.

\footnotetext{
*Endereço de correspondência: marcel.novaes@gmail.com
}

Nossa afirmação inicial, que pode ter parecido surpreendente à primeira vista, acabou se revelando bastante simples (como muitas vezes ocorre no contexto da probabilidade). Mas o experimento imaginário envolvido sugere questões interessantes: Qual a chance de que as posições inicial e final de um ponto estejam mais distantes que um certo valor? Qual a chance de que a posição final do ponto $x$ esteja próxima do ponto $y$ ? Qual a chance de que todos os pontos da bola voltem à mesma posição?

O que estamos tentando motivar é o estudo de um conceito que não se costuma abordar nas aulas de estatística, o de rotações aleatórias. Fisicamente, está claro o que queremos: pegamos uma bola e a chutamos ou jogamos para cima e, quando a apanharmos de volta, ela terá sofrido uma rotação que não sabemos qual é e portanto podemos dizer que é aleatória. Mas é claro que matematicamente a situação é um pouco mais difícil. Afinal, uma rotação não é um número, e sim um operador, e a ideia de operadores aleatórios é mais sofisticada que a ideia de números aleatórios. Então, o que é exatamente uma rotação aleatória? Quais as propriedades dessa criatura? Isso serve para alguma coisa?

A última pergunta talvez tenha sido a primeira a passar pela cabeça do leitor. A resposta é simples: processos aleatórios são sempre úteis. Isso é verdade porque os fenômenos naturais costumam ser extremamente complicados e nós normalmente não somos capazes de descrevê-los em todos os seus detalhes, de modo que pode ser vantajoso utilizar descrições estatísticas. Como 
não somos capazes de prever qual número o dado que lançamos vai mostrar, então dizemos que cada lado tem $1 / 6$ de chance e pronto. Se um sistema sofre uma rotação complicada que não sabemos descrever, consideramos que ela seja aleatória. Se não estamos preocupados com a orientação de um objeto, podemos calcular um valor médio integrando sobre todas as rotações que ele pode sofrer. Além disso, as rotações estão entre os mais simples dos operadores e entender sua versão aleatória pode nos ajudar a entender a versão aleatória de operadores [1], que aparecem em estatística multivariada [2] e em temas mais aplicados como, por exemplo, no estudo de desordem em materiais [3, em fenômenos caóticos [4] ou em engenharia mecânica [5]. A ideia de aleatoriedade pode operar o milagre de transformar o complicado em simples.

Este artigo está organizado da seguinte maneira. Começamos falando um pouco sobre o grupo de rotações na Seção 2. Depois, abordamos o problema de como sortear rotações aleatórias no computador, na Seção 3 e aproveitamos para mostrar alguns resultados numéricos. Nas seções 4 e 5 damos atenção a duas questões fundamentais sobre rotações aleatórias: a distribuição dos seus elementos de matriz e a distribuição dos seus autovalores. Apresentamos conclusões na Seção 6 .

\section{O grupo de rotações e o grupo ortogonal}

\subsection{No plano $\mathbb{R}^{2}$}

Começamos com o plano $\mathbb{R}^{2}$, com a coordenada horizontal denotada por $x$ e a vertical por $y$, sendo $\left(\begin{array}{ll}1 & 0\end{array}\right)$ e $\left(\begin{array}{ll}0 & 1\end{array}\right)$ os versores ortogonais correspondentes. Uma rotação anti-horária por um ângulo $\theta$, digamos $R_{\theta}$, age sobre os versores de acordo com

$$
R_{\theta}(10)=(\cos \theta \sin \theta), \quad R_{\theta}(01)=(-\sin \theta \cos \theta)
$$

e portanto pode ser representada, na base desses versores, pela matriz

$$
R_{\theta}=\left(\begin{array}{cc}
\cos \theta & -\sin \theta \\
\sin \theta & \cos \theta
\end{array}\right), \quad 0 \leq \theta<2 \pi .
$$

O quadrado da distância entre dois pontos é o módulo quadrado do vetor entre eles, ou seja, é o produto escalar do vetor consigo mesmo,

$$
\left(\begin{array}{ll}
x & y
\end{array}\right)\left(\begin{array}{l}
x \\
y
\end{array}\right)=x^{2}+y^{2}
$$

Rotações preservam distância: o módulo quadrado do vetor rodado é

$$
\left(\begin{array}{ll}
x & y
\end{array}\right) R_{\theta}^{T} R_{\theta}\left(\begin{array}{l}
x \\
y
\end{array}\right)=\left(\begin{array}{ll}
x & y
\end{array}\right)\left(\begin{array}{l}
x \\
y
\end{array}\right)
$$

onde usamos a operação de transposição para passar de um vetor coluna a um vetor linha e aplicamos a identidade $R_{\theta}^{T} R_{\theta}=1$, que pode ser deduzida diretamente de 2 .
Se uma matriz qualquer $O$ satisfaz a condição $O^{T} O=1$, ela é dita ortogonal. O conjunto de todas as matrizes ortogonais em duas dimensões é chamado de Grupo Ortogonal e denotado por $\mathcal{O}(2)$. É evidente que esse conjunto é um grupo: o produto de duas matrizes ortogonais é também uma matriz ortogonal, a inversa de uma matriz ortogonal é ortogonal, a identidade é uma matriz ortogonal e o produto é associativo (essas são as condições que definem um grupo).

Como vimos, rotações podem ser representadas por matrizes ortogonais. Será que toda matriz ortogonal é uma rotação? A resposta é não. As matrizes $\left(\begin{array}{cc}1 & 0 \\ 0 & -1\end{array}\right)$ e $\left(\begin{array}{cc}-1 & 0 \\ 0 & 1\end{array}\right)$ são ortogonais, mas não representam nenhuma rotação. Em vez disso, elas representam reflexões em relação aos eixos horizontal e vertical, respectivamente. Naturalmente, podemos definir reflexões em relação a qualquer eixo do plano. A matriz associada à reflexão em relação ao eixo definido pelo vetor $(\cos (\theta) \sin (\theta))$ é dada por (é uma pena que as palavras 'rotação' e 'reflexão' comecem com a mesma letra):

$$
\Re_{\theta}=\left(\begin{array}{cc}
\cos (2 \theta) & \sin (2 \theta) \\
\sin (2 \theta) & -\cos (2 \theta)
\end{array}\right), \quad 0 \leq \theta<\pi .
$$

(note que o domínio da variável $\theta$ neste caso vai só até $\pi$, pois estamos especificando apenas a direção e não o sentido do eixo).

O conjunto das rotações é um subgrupo (a composição de duas rotações é uma rotação, $R_{\theta} R_{\phi}=R_{\theta+\phi}$; toda rotação possui uma inversa, $R_{\theta}^{-1}=R_{-\theta}$; a identidade é $R_{0}$ ) do grupo ortogonal, chamado de Grupo Ortogonal Especial ou $\mathcal{S O}(2)$. Por outro lado, o conjunto que contém somente as reflexões não forma um grupo porque a composição de duas reflexões não é uma reflexão e sim uma rotação:

$$
\Re_{\theta} \Re_{\phi}=R_{2 \theta-2 \phi} .
$$

O grupo $\mathcal{O}(2)$ consiste portanto das rotações e das reflexões (note que a inversão $(x y) \rightarrow(-x-y)$ é uma rotação de $\pi$ ). O determinante de uma matriz em $\mathcal{O}(2)$ só pode ser igual a 1 ou a -1 , pois a condição $O^{T} O=1$ implica $(\operatorname{det} O)^{2}=1$. As reflexões têm determinante negativo, pois alteram a orientação de uma das componentes do vetor em que atuam, enquanto que as rotações têm determinante positivo. Assim, o subgrupo $\mathcal{S O}(2)$ pode ser especificado, dentro do grupo $\mathcal{O}(2)$, como contendo as matrizes $O$ tais que $\operatorname{det} O=1$.

Com relação a autovalores e autovetores, os dois tipos de operação são complementares. No caso das rotações $R_{\theta}$, os autovalores são $e^{i \theta}$ e $e^{-i \theta}$ e dependem do ângulo, enquanto os autovetores são fixos, $\left(\begin{array}{l}i \\ 1\end{array}\right)$ e $\left(\begin{array}{c}-i \\ 1\end{array}\right)$, respectivamente. Já no caso das reflexões $\Re_{\theta}$, os autovalores é que são fixos, sempre 1 e -1 , enquanto os autovetores dependem do ângulo, $\left(\begin{array}{c}\cos \theta \\ \sin \theta\end{array}\right)$ e $\left(\begin{array}{c}-\sin \theta \\ \cos \theta\end{array}\right)$, respectivamente. 
Se quisermos rotações e reflexões aleatórias no plano, basta considerarmos $\theta$ uma variável aleatória. Podemos tomar uma distribuição uniforme no intervalo $(0,2 \pi)$ para as rotações e no intervalo $(0, \pi)$ para as reflexões. Nesse contexto, três problemas são muito naturais: entender a estatística dos autovalores, dos autovetores e dos elementos de matriz.

No caso dos autovalores, faz mais sentido olhar para as rotações. Como as matrizes são reais, eles existem sempre em pares conjugados e estão uniformemente distribuídos sobre o círculo unitário no plano complexo. No caso dos autovetores, faz mais sentido olhar para as reflexões. Vemos que eles são ortogonais e também estão uniformemente distribuídos sobre o círculo unitário em $\mathbb{R}^{2}$.

Quanto se trata dos elementos de matriz, é mais conveniente tratar o grupo $\mathcal{O}(2)$ inteiro, entendendo que

$$
\langle f(O)\rangle_{\mathcal{O}(2)}=\frac{1}{4 \pi} \int_{0}^{2 \pi} f\left(R_{\theta}\right) d \theta+\frac{1}{2 \pi} \int_{0}^{\pi} f\left(\Re_{\theta}\right) d \theta
$$

É fácil concluir que o valor médio de todos os elementos de matriz é zero $\left\langle O_{i j}\right\rangle_{O(2)}=0$. Com relação às correlações entre os elementos, temos:

$$
\left\langle(O)_{i_{1} j_{1}}(O)_{i_{2} j_{2}}\right\rangle_{\mathcal{O}(2)}=\left\{\begin{array}{l}
\frac{1}{2}, \text { se } i_{1}=i_{2} \text { e } j_{1}=j_{2}, \\
0, \text { caso contrário. }
\end{array}\right.
$$

(convidamos o leitor a verificar esta afirmação). Vemos que os elementos estão descorrelacionados, o que não seria verdade se olhássemos só para as rotações ou só para as reflexões.

\subsection{No espaço $\mathbb{R}^{N}$}

O grupo ortogonal $\mathcal{O}(N)$ é definido como o conjunto das matrizes que representam transformações lineares que preservam o produto escalar,

$$
\langle\mathbf{x} \mid \mathbf{y}\rangle=\sum_{k=1}^{N} x_{k} y_{k}
$$

ou seja, das matrizes reais $N \times N$ que satisfazem

$$
O^{T} O=O O^{T}=I_{N}
$$

onde $I_{N}$ é a matriz identidade. Para uma discussão pormenorizada sobre o grupo ortogonal e as rotações, incluindo uma interessante introdução aos quatérnions, sugerimos a leitura de [6]. Para o caso particular $N=3$ e sua conexão com a teoria do momento angular em mecânica quântica, recomendamos [7].

Assim como no caso $N=2$, o grupo $\mathcal{O}(N)$ possui duas componentes distintas: as rotações, que têm determinante +1 e formam o subgrupo $\mathcal{S O}(N)$, e as reflexões, que têm determinante -1 e não formam um subgrupo (o produto de duas reflexões não é uma reflexão). Essas duas componentes são como imagens no espelho uma da outra e existe uma bijeção simples entre elas. Se tomarmos uma reflexão qualquer, por exemplo a matriz diagonal cujo primeiro elemento é -1 e todos os outros são 1 , e multiplicarmos todos os elementos de $\mathcal{S O}(N)$ por essa reflexão, obteremos justamente o conjunto das reflexões. Em marcado contraste com o caso $N=2$, para todo $N>2$ o grupo das rotações não é comutativo.

No caso $N=3$ toda rotação se dá em torno de um eixo, que permanece invariante. Para $N>3$ é interessante introduzir a noção de rotação elementar. Uma rotação é elementar se afeta apenas um espaço de dimensão 2. Toda rotação em $\mathcal{S O}(2)$ ou em $\mathcal{S O}(3)$ é elementar, mas isso não é mais verdade para $N>3$. Por exemplo, toda matriz em $\mathcal{S O}(4)$ pode ser escrita, por meio de uma mudança de base, na forma

$$
\left(\begin{array}{cccc}
\cos \theta & -\sin \theta & 0 & 0 \\
\sin \theta & \cos \theta & 0 & 0 \\
0 & 0 & \cos \phi & -\sin \phi \\
0 & 0 & \sin \phi & \cos \phi
\end{array}\right)
$$

ou seja, como duas rotações elementares independentes. Note que não se pode falar em eixo de rotação neste caso. Essa estrutura se repete em dimensões mais altas. Todo elemento de $\mathcal{S O}(5)$, por exemplo, consiste de duas rotações elementares e uma direção invariante. Resumindo, os elementos de $\mathcal{S O}(2 N)$ consistem de $N$ rotações elementares; essas matrizes possuem $N$ pares de autovalores conjugados (que podem ser iguais a 1 caso haja rotações elementares triviais). Por outro lado, elementos de $\mathcal{S O}(2 N+1)$ consistem de $N$ rotações elementares e uma direção invariante; nesse caso, pelo menos um dos autovalores será sempre igual a 1.

Também no caso das reflexões é interessante introduzir a ideia das operações elementares. Uma reflexão elementar age sobre $\mathbb{R}^{N}$ mantendo um espaço $\mathbb{R}^{N-1}$ invariante e trocando o sentido de um espaço unidimensional. Essas matrizes sempre têm portanto $N-1$ autovalores iguais a 1 e um autovalor igual a -1 . A composição de um número par de reflexões elementares é uma rotação, e a composição de um número ímpar é uma reflexão, que não será elementar se o autovalor -1 for degenerado. De fato, um teorema de Cartan garante que qualquer elemento de $\mathcal{O}(N)$ pode sempre ser escrito como o produto de no máximo $N$ reflexões (ver, por exemplo, o capítulo 8 de [8]).

Como podemos fazer para considerar rotações aleatórias em $\mathbb{R}^{N}$ ? Uma possibilidade é escrever as matrizes explicitamente em termos de algumas variáveis, tais como o ângulo $\theta$ no caso do $\mathbb{R}^{2}$ ou os ângulos de Euler no caso do $\mathbb{R}^{3}$, e depois tomar essas variáveis de forma aleatória. Mas como são as matrizes? Antes de mais nada, quantas variáveis precisamos? Essa é a dimensão do grupo. Uma matriz $N \times N$ possui $N^{2}$ elementos, porém o vínculo 10 garante que não são todos independentes.

A melhor maneira de encontrar a dimensão do grupo $\mathcal{S O}(N)$ é expressar seus elementos na forma $O=e^{X}$ 
para algum $X$, sendo a exponencial definida simplesmente em termos da série de Taylor,

$$
e^{X}=I_{N}+X+\frac{X^{2}}{2 !}+\frac{X^{3}}{3 !}+\cdots
$$

Para a matriz $X$, a condição de vínculo $O^{T}=O^{-1}$ se traduz em $X^{T}=-X$, de modo que $X$ deve ser antisimétrica. Essa é a única restrição que devemos impor a $X$. Assim, seus elementos diagonais são todos nulos e os elementos abaixo da diagonal são os negativos dos elementos acima da diagonal. Cada elemento acima da diagonal é um grau de liberdade, ou seja contribui para a dimensão do grupo, que portanto é $N(N-1) / 2$. A relação $e^{\operatorname{Tr} X}=\operatorname{det} O$ mostra que $\operatorname{det} O=1$, por isso esse procedimento permite a construção apenas das rotações e não das reflexões.

Enquanto $\mathcal{S O}(N)$ é um grupo de Lie, o conjunto das matrizes reais anti-simétricas forma uma álgebra de Lie. Para uma introdução a essa teoria, recomendamos o livro de Stillwell [9].

Então sabemos que são necessárias $N(N-1) / 2$ variáveis para parametrizarmos $\mathcal{S O}(N)$ ou $\mathcal{O}(N)$. Mas qual é a cara das matrizes? Elas são relativamente complicadas e, por simplicidade, preferimos omitir essa discussão. A construção explícita pode ser encontrada por exemplo em [10] ou em [11]. Em vez de usarmos essas parametrizações, vamos recorrer a argumentos mais gerais.

\section{Resultados Numéricos}

No grupo ortogonal, assim como em outros grupos de matrizes, existe uma medida de probabilidade natural, conhecida como medida de Haar. Vamos denotá-la por $P(O)$. No caso do $\mathcal{O}(N)$, essa medida é invariante por multiplicação tanto à esquerda quanto à direita, ou seja, $P(O A)=P(A O)=P(O)$ para qualquer $A$ pertencente ao grupo. Isso quer dizer simplesmente que a medida é uniforme e que todos os elementos do grupo são igualmente prováveis em um sorteio. A medida de Haar sobre os grupos de matrizes é análoga à distribuição uniforme sobre a circunferência ou sobre qualquer intervalo finito.

Como podemos, então, sortear uma matriz de rotação distribuída segundo a medida de Haar? Bom, as colunas de $O$ são $N$ vetores ortonormais. Precisamos então construir $N$ vetores ortonormais aleatórios. Isso pode ser feito aplicando o processo de ortogonalização de Gram-Schmidt aos autovetores de alguma outra matriz aleatória, com elementos independentes e gaussianos, como descrito por Mezzadri [12. Esse algoritmo está implementado na maioria das linguagens de programação e é chamado decomposição $Q R$ : ele escreve uma matriz qualquer como o produto de uma matriz ortogonal, $Q$, e uma matriz triangular superior, $R$.

Entretanto, a decomposição QR não é única, pois basta trocar $Q$ por $Q A$ e $R$ por $A^{-1} R$ para obter outra decomposição equivalente. Para torná-la única, impomos que os elementos não nulos da diagonal principal de $R$ devem ser positivos. Com isso, a matriz $Q$ terá exatamente a distribuição de Haar sobre o grupo $\mathcal{O}(N)$. O código a seguir implementa esse algoritmo em Python:

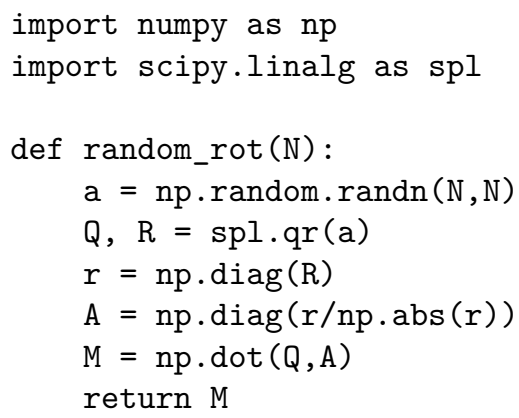

$\mathrm{O}$ algoritmo começa sorteando uma matriz a com distribuição normal. Depois, aplica a decomposição QR. Os elementos diagonais da matriz $R$ são colocados no vetor $r$ e seus sinais são colocados na matriz diagonal $A$. Finalmente, a matriz ortogonal que havia resultado da decomposição QR é multiplicada por $A$ pela direita, garantindo unicidade.

Se quisermos o grupo $\mathcal{S O}(N)$ em vez do $\mathcal{O}(N)$, podemos simplesmente calcular o determinante da matriz $O$ e descartá-la se este for -1 , ou então podemos multiplicar $O$ por uma reflexão fixa para transformá-la numa rotação.

Agora que somos capazes de gerar matrizes aleatórias distribuídas uniformemente sobre o grupo $\mathcal{O}(N)$ de acordo com a medida de Haar, podemos investigar numericamente suas propriedades estatísticas. Comecemos com seus autovalores.

Eles são uniformemente distribuídos no círculo unitário, conforme vemos na Fig. 1p, onde mostramos os autovalores de uma única matriz ortogonal aleatória de ordem 100. Entretanto, é possível notar que os autovalores não são variáveis independentes. Basta comparar com a Fig. 17a, onde temos 100 pontos aleatórios independentes sobre o círculo. Esses pontos tendem a formar aglomerados, em contraste com os autovalores, que preferem manter distância entre si. Este comportamento é
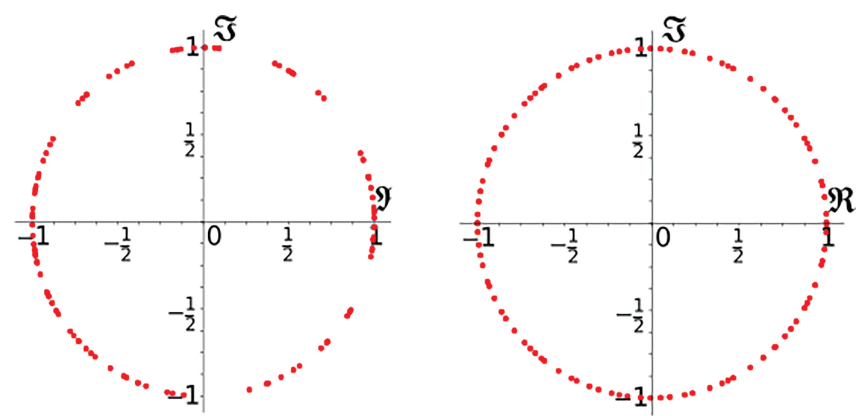

Figura 1: Comparação entre 100 pontos independentes distribuídos no círculo unitário (à esquerda) e o autovalores de uma matriz de rotação de ordem 100 (à direita). Podemos detectar que há repulsão entre os autovalores, que têm uma distribuição mais uniforme. 
chamado de repulsão. Os autovalores comportam-se mais ou menos como cargas de mesmo sinal dispostas sobre o círculo, ou seja, tendem a se afastar uns dos outros. Veremos isso com mais detalhes a seguir.

Como os autovalores têm módulo 1, podemos escrevêlos como $e^{i \theta} \operatorname{com} \theta \in[0,2 \pi)$. A distribuição dessas autofases encontra-se na Fig. 2 a (sorteamos $10^{5}$ matrizes de ordem $N=100$ ). Encontramos de fato uma distribuição aproximadamente uniforme (igual a $\frac{1}{2 \pi}$ para normalização), exceto por uma ligeira prevalência das fases $\theta=0$ e $\theta=\pi$, associadas aos autovalores \pm 1 , mas esse efeito diminui conforme $N$ se torna maior.

Uma quantidade de destaque no estudo de matrizes é o traço, a soma dos elementos da diagonal principal ou a soma dos autovalores. A distribuição do traço encontra-se na Fig. 22 (calculada usando as mesmas $10^{5}$ matrizes de ordem $N=100)$. Como o traço é a soma de um grande número de variáveis aleatórias, ainda que não independentes, poderíamos suspeitar que tivesse distribuição normal, devido a um processo semelhante ao Teorema Central do Limite. Vemos que sua distribuição é de fato bem aproximada por uma distribuição normal com média zero e desvio padrão 1 . Na Seção 4.3 veremos que a distribuição é realmente essa e não depende do tamanho das matrizes.

Também podemos nos perguntar sobre a distribuição dos elementos de matriz. Na Fig. 3 temos a distribuição de um elemento da diagonal principal, $O_{11}$, e de um elemento fora da diagonal, $O_{34}$. A distribuição dos elementos parece não depender dos índices. Entretanto,
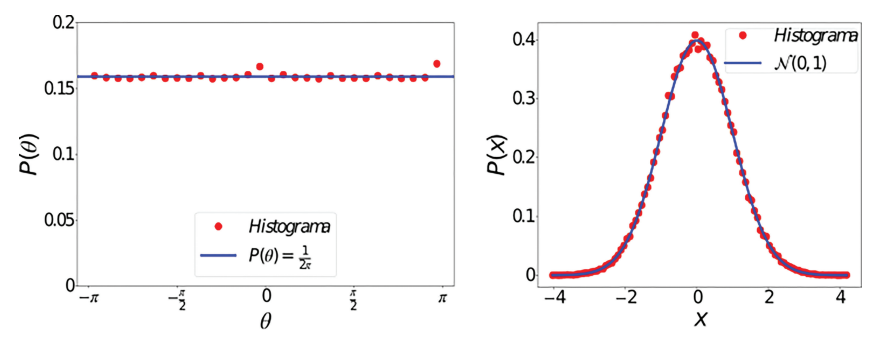

Figura 2: À esquerda, distribuição das fases dos autovalores de matrizes ortogonais. À direita, distribuição do traço, $x=\operatorname{Tr} O$. Nos dois casos, sorteamos $10^{5}$ matrizes de dimensão 100 .
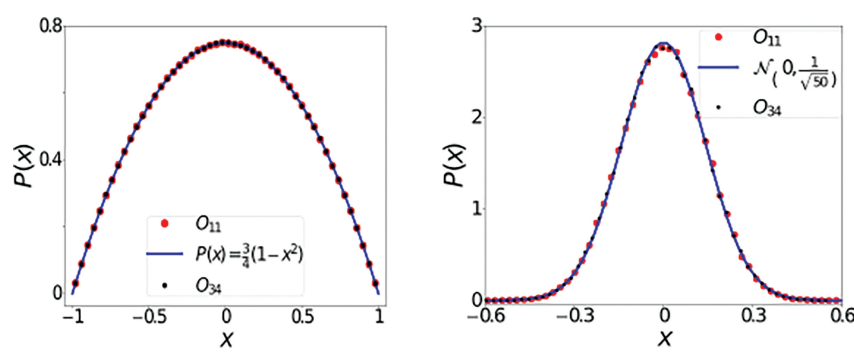

Figura 3: Distribuição dos elementos de matriz: $O_{11}$ em vermelho e $O_{34}$ em preto. À esquerda, as matrizes têm dimensão $N=5$ e a curva azul é o resultado exato. À direita, $N=50$ e a curva azul é o resultado assintótico, gaussiano. depende da ordem das matrizes. À esquerda, temos a distribuição para matrizes de ordem $N=5$; à direita, matrizes de ordem $N=50$. Conforme o tamanho das matrizes aumenta, a distribuição se aproxima cada vez mais de uma distribuição normal com média zero e desvio padrão $1 / \sqrt{N}$.

\section{Elementos de Matriz}

Como os elementos de uma matriz ortogonal podem ser positivos ou negativos com igual probabilidade, é natural que todos tenham valor esperado nulo, $\left\langle O_{i j}\right\rangle_{\mathcal{O}(N)}=0$. E a variância? Como a distribuição das matrizes é invariante por rotação, todos os elementos devem ter a mesma variância. A condição $O^{T} O=1$ é equivalente a $\sum_{j=1}^{N} O_{i j}^{2}=1$ para todo $i$, de modo que podemos concluir que

$$
\left\langle O_{i j}^{2}\right\rangle_{\mathcal{O}(N)}=\frac{1}{N}
$$

\subsection{Dimensão grande}

E quanto às correlações entre os elementos de matriz? Eles não podem ser independentes, pois as colunas da matriz $O$ devem ser ortogonais e normalizadas.

Olhemos apenas uma das colunas, esquecendo a ortogonalidade e levando em conta somente a normalização. Temos então um vetor $\vec{x}$ de dimensão $N$, normalizado, $\sum_{i=1}^{N} x_{i}^{2}=1$, e queremos saber qual é a distribuição conjunta dos primeiros $m$ elementos. Se o único vínculo que as variáveis precisam satisfazer é a normalização, então essa distribuição é proporcional a

$$
\int d x_{m+1} \cdots d x_{N} \delta\left(1-\sum_{i=1}^{N} x_{i}^{2}\right)
$$

Usando a representação integral da distribuição delta, $\delta(x) \propto \int e^{i k x} d k$, temos

$$
\int d k e^{i k\left(1-\sum_{i=1}^{m} x_{i}^{2}\right)}\left(\int d x e^{-i k x^{2}}\right)^{N-m}
$$

que é proporcional a

$$
\int d k e^{i k\left(1-\sum_{i=1}^{m} x_{i}^{2}\right)} k^{-(N-m) / 2} \propto\left(1-\sum_{i=1}^{m} x_{i}^{2}\right)^{(N-m) / 2-1} .
$$

Em particular, a distribuição de um único elemento é proporcional a $\left(1-x^{2}\right)^{(N-3) / 2}$. Essa é a distribuição que aparece na Figura 3a. Note que a variável reescalada $y=x \sqrt{N}$ adquire distribuição normal para $N$ grande, pois $\left(1-y^{2} / N\right)^{N} \approx e^{-y^{2}}$.

De forma mais geral, podemos olhar para um bloco de elementos de matriz. Seja $u$ o bloco que contém os elementos $O_{i j}$ com $i, j \leq m$. Então, $u$ é uma matriz aleatória e sua distribuição é conhecida [13]: ela é proporcional a

$$
\operatorname{det}\left(1-u u^{T}\right)^{(N-1) / 2-m}
$$


Note que este resultado é análogo a $(16)$. Novamente, se $N \gg 1$ então a matriz renormalizada $v=u \sqrt{N}$ tem distribuição normal, pois $\left(1-v v^{T} / N\right)^{(N-1) / 2-m} \approx e^{-v v^{T}}$. Concluímos que se a dimensão $N$ for muito grande, o vínculo da ortogonalidade não é muito forte e os elementos de matriz se tornam variáveis aleatórias aproximadamente independentes.

\subsection{Dimensão finita, exemplos}

Podemos dizer mais sobre a distribuição dos elementos de matriz, mesmo para dimensões $N$ finitas. É possível mostrar, por exemplo, que [14]

$$
\left\langle O_{11}^{4}\right\rangle_{\mathcal{O}(N)}=\frac{3}{N(N+2)}
$$

e que 14

$$
\left\langle O_{11}^{2} O_{22}^{2}\right\rangle_{\mathcal{O}(N)}=\frac{(N+1)}{N(N-1)(N+2)} .
$$

Vemos que, para $N$ grande, este último resultado se aproxima de $\left\langle O_{11}^{2}\right\rangle_{\mathcal{O}(N)}\left\langle O_{22}^{2}\right\rangle_{\mathcal{O}(N)}$ (ver $(13)$ ), como deveria em vista da independência assintótica. Como último exemplo, temos

$$
\left\langle O_{14} O_{12} O_{32} O_{34}\right\rangle_{\mathcal{O}(N)}=\frac{-1}{N(N-1)(N+2)} .
$$

Note que este resultado é de ordem $1 / N$ vezes menor que o de cima, refletindo também a independência assintótica das variáveis para $N$ grande.

\subsection{Dimensão finita, teoria}

Vamos chamar de pareamentos as partições do conjunto $\{1,2, \ldots, 2 n\}$ em pares, ou seja, em $n$ blocos de tamanho 2. Expressamos um pareamento $\mathfrak{m}$ na forma $\{\{\mathfrak{m}(1), \mathfrak{m}(2)\},\{\mathfrak{m}(3), \mathfrak{m}(4)\}, \ldots\}$. Por exemplo, no caso de quatro elementos, existem três pareamentos,

$$
\begin{gathered}
\mathfrak{m}_{1}=\{\{1,2\},\{3,4\}\}, \quad \mathfrak{m}_{2}=\{\{1,3\},\{2,4\}\}, \\
\mathfrak{m}_{3}=\{\{1,4\},\{2,3\}\} .
\end{gathered}
$$

Seja $\mathcal{M}_{n}$ o conjunto dos pareamentos possíveis em um conjunto de $2 n$ números. O tamanho desse conjunto é $(2 n-1) ! !=(2 n-1)(2 n-3)(2 n-5) \ldots$

Dada uma lista de números, digamos $\left(i_{1}, \ldots, i_{2 n}\right)$, dizemos que essa lista satisfaz o pareamento $\mathfrak{m}$ se seus elementos coincidem de acordo com $\mathfrak{m}$. Ou seja, se $i_{\mathfrak{m}(2 k-1)}=i_{\mathfrak{m}(2 k)}$ para todo $k$. Vejamos alguns exemplos. A lista $a=(1,1,2,2)$ satisfaz $\mathfrak{m}_{1}$ porque

$$
\begin{aligned}
& a_{\mathfrak{m}_{1}(1)}=a_{1}=a_{\mathfrak{m}_{1}(2)}=a_{2}=1, \\
& a_{\mathfrak{m}_{1}(3)}=a_{3}=a_{\mathfrak{m}_{1}(4)}=a_{4}=2 .
\end{aligned}
$$

Por outro lado, a lista $b=(1,2,1,2)$ satisfaz $\mathfrak{m}_{2}$ porque

$$
\begin{aligned}
& b_{\mathfrak{m}_{2}(1)}=b_{1}=b_{\mathfrak{m}_{2}(2)}=b_{3}=1 \\
& b_{\mathfrak{m}_{2}(3)}=b_{2}=b_{\mathfrak{m}_{2}(4)}=b_{4}=2 .
\end{aligned}
$$

Finalmente, a lista $c=(1,2,2,1)$ satisfaz $\mathfrak{m}_{3}$ porque

$$
\begin{aligned}
& c_{\mathfrak{m}_{3}(1)}=c_{1}=c_{\mathfrak{m}_{3}(2)}=c_{4}=1, \\
& c_{\mathfrak{m}_{3}(3)}=c_{2}=c_{\mathfrak{m}_{3}(4)}=c_{3}=2 .
\end{aligned}
$$

A lista $(1,1,1,1)$ satisfaz todos os pareamentos.

Por que estamos falando em pareamentos? Porque a integral

$$
\left\langle\prod_{k=1}^{2 n} O_{i_{k} j_{k}}\right\rangle_{\mathcal{O}(N)}
$$

só é diferente de zero se a lista de números $\left(i_{1}, \ldots, i_{2 n}\right)$ satisfizer algum pareamento, e o mesmo para a lista $\left(j_{1}, \ldots, j_{2 n}\right)$. Em termos concretos, se $\Delta_{\mathfrak{m}}(i)$ é a função que vale 1 quando a lista $i$ satisfaz o pareamento $\mathfrak{m}$ e zero caso contrário, então a integral pode ser escrita assim:

$$
\left\langle\prod_{k=1}^{2 n} O_{i_{k} j_{k}}\right\rangle_{\mathcal{O}(N)}=\sum_{\mathfrak{m}, \mathfrak{n} \in \mathcal{M}_{n}} \Delta_{\mathfrak{m}}(i) \Delta_{\mathfrak{n}}(j) W(\mathfrak{m}, \mathfrak{n}) .
$$

Por exemplo, podemos notar que as listas $i=$ $(1,1,3,3)$ e $j=(4,2,2,4)$ aparecem no exemplo 20 e portanto, de acordo com a fórmula acima, temos que o lado direito da equação 20 é igual a $W\left(\mathfrak{m}_{1}, \mathfrak{m}_{3}\right)$.

Resta dizer quem é a função $W$, chamada de função de Weingarten. Para isso, definimos um grafo $\Gamma(\mathfrak{m}, \mathfrak{n})$ da seguinte maneira: temos vértices com índices de 1 a $2 n$ e ligamos dois vértices se seus índices aparecem no mesmo bloco em $\mathfrak{m}$ ou em $\mathfrak{n}$. Depois, definimos uma matriz $G$ cujos elementos são dados por $G_{\mathfrak{m}, \mathfrak{n}}=N^{c(\mathfrak{m}, \mathfrak{n})}$, onde $c$ é o número de caminhos fechados no grafo $\Gamma(\mathfrak{m}, \mathfrak{n})$. Finalmente, a função $W(\mathfrak{m}, \mathfrak{n})$ é igual [14 ao elemento $(\mathfrak{m}, \mathfrak{n})$ da inversa da matriz $G$.

Por exemplo, para $n=2$ temos os três pareamentos em (21). A matriz $G$ nesse caso é

$$
G=\left(\begin{array}{ccc}
N^{2} & N & N \\
N & N^{2} & N \\
N & N & N^{2}
\end{array}\right)
$$

e sua inversa é

$$
G^{-1}=\frac{1}{N(N-1)(N+2)}\left(\begin{array}{ccc}
N+1 & -1 & -1 \\
-1 & N+1 & -1 \\
-1 & -1 & N+1
\end{array}\right) .
$$

De fato, o elemento $\left(G^{-1}\right)_{13}$ dessa matriz coincide com o lado direito de 20. Por outro lado, no cálculo de $\left\langle O_{11}^{4}\right\rangle_{\mathcal{O}(N)}$ todos os pareamentos são possíveis, e o resultado é igual à soma de todos os elementos de $G^{-1}$.

(O leitor atento terá percebido que a matriz acima não está definida para $N=1$. A matriz $G$ associada aos pareamentos de $2 n$ números de fato não tem inversa se $N<n$. Nesse caso é preciso usar a pseudo-inversa [15]. Omitimos essa discussão por simplicidade).

Como aplicação desta teoria, podemos considerar a distribuição do traço da matriz. Vamos abordar esse 
problema usando o método dos momentos, ou seja, calculando $\left\langle(\operatorname{Tr} O)^{2 n}\right\rangle_{\mathcal{O}(N)}$ para todo $n$ (potências ímpares do traço têm média zero). Usando a fórmula (26), temos

$$
\left\langle(\operatorname{Tr} O)^{2 n}\right\rangle_{\mathcal{O}(N)}=\sum_{i_{1}, \ldots, i_{2 n}} \sum_{\mathfrak{m}, \mathfrak{n} \in \mathcal{M}_{n}} \Delta_{\mathfrak{m}}(i) \Delta_{\mathfrak{n}}(i) W(\mathfrak{m}, \mathfrak{n})
$$

(note que a lista $j$ é igual à lista $i$ neste caso). Trocando a ordem das somas, temos a quantidade $\sum_{i_{1}, \ldots, i_{2 n}} \Delta_{\mathfrak{m}}(i) \Delta_{\mathfrak{n}}(i)$. As funções $\Delta$ requerem que algumas das variáveis $i$ sejam iguais e o resultado da soma será $N$ elevado ao número de variáveis independentes. Alguma reflexão nos convencerá de que esse número é exatamente $c(\mathfrak{m}, \mathfrak{n})$. Assim, ficamos com

$$
\left\langle(\operatorname{Tr} O)^{2 n}\right\rangle_{\mathcal{O}(N)}=\sum_{\mathfrak{m}, \mathfrak{n} \in \mathcal{M}_{n}} G_{\mathfrak{m}, \mathfrak{n}} W(\mathfrak{m}, \mathfrak{n})
$$

Mas definimos $W(\mathfrak{m}, \mathfrak{n})$ justamente como os elementos de matriz da inversa de $G$, de modo que o resultado se reduz $\mathrm{a}\left\langle(\operatorname{Tr} O)^{2 n}\right\rangle_{\mathcal{O}(N)}=\sum_{\mathfrak{m} \in \mathcal{M}_{n}} 1=(2 n-1)$ !!. Esses são os momentos de uma variável aleatória com distribuição normal (esta conta pressupõe a existência de $G^{-1}$, portanto só vale se $N>2 n$, de modo que a distribuição só se aproxima da normal para grandes valores de $N$ ).

Existe uma fórmula mais explícita para a função de Weingarten, que está relacionada ao fato de que pareamentos podem ser vistos como representantes das classes laterais do grupo do hiperoctaedro em relação ao grupo de permutações $S_{2 n}$. Preferimos omitir essa discussão, que pode ser encontrada em [14, por ser muito técnica.

\section{Autovalores}

\subsection{Distribuição conjunta}

Como já vimos, os autovalores das reflexões são fixos (sempre iguais a 1 ou -1). Portanto, para discutir autovalores restringimos a discussão ao subgrupo das rotações, o $\mathcal{S O}(N)$. Além disso, é preciso distinguir os casos de dimensão par e os de dimensão ímpar. No segundo caso um dos autovalores é sempre igual a 1. Tanto para $\mathcal{S O}(2 N)$ quanto para $\mathcal{S O}(2 N+1)$, os (demais) autovalores são denotados por $e^{i \theta_{k}}$ e $e^{-i \theta_{k}}$, com $1 \leq k \leq N$. Os valores $0 \leq \theta_{k} \leq \pi$ são as autofases.

A distribuição de probabilidade conjunta das autofases foi obtida por H. Weyl [16], sendo dada por

$$
P_{\mathcal{S O}(2 N)}\left(\theta_{1}, \ldots, \theta_{N}\right) \propto \prod_{1 \leq j<k \leq N}\left(\cos \left(\theta_{k}\right)-\cos \left(\theta_{j}\right)\right)^{2}
$$

em dimensão par e por

$$
\begin{aligned}
& P_{\mathcal{S O}(2 N+1)}\left(\theta_{1}, \ldots, \theta_{N}\right) \\
& \propto \prod_{j=1}^{N} \sin ^{2}\left(\theta_{j} / 2\right) \prod_{1 \leq j<k \leq N}\left(\cos \left(\theta_{k}\right)-\cos \left(\theta_{j}\right)\right)^{2}
\end{aligned}
$$

$$
\propto \prod_{j=1}^{N} \sin ^{2}\left(\theta_{j} / 2\right) \prod_{1 \leq j<k \leq N}\left(\cos ^{2}\left(\theta_{k} / 2\right)-\cos ^{2}\left(\theta_{j} / 2\right)\right)^{2}
$$

em dimensão ímpar.

Vemos que os autovalores são variáveis aleatórias correlacionadas e podemos enxergar o seu comportamento repulsivo, já que as distribuições se anulam quadraticamente quando dois deles são próximos, $\theta_{k} \approx \theta_{j}$, de modo que degenerescências são improváveis.

Se no caso da dimensão par denotarmos $\cos \left(\theta_{j}\right)=x_{j}$, a distribuição fica sendo

$$
\begin{aligned}
& P_{\mathcal{S O}(2 N)}\left(x_{1}, \ldots, x_{N}\right) \\
& \propto \prod_{j=1}^{N}\left(1-x_{j}^{2}\right)^{-1 / 2} \prod_{1 \leq j<k \leq N}\left(x_{k}-x_{j}\right)^{2},
\end{aligned}
$$

com $-1 \leq x_{j} \leq 1$. Analogamente, se no caso da dimensão ímpar denotarmos $\cos ^{2}\left(\theta_{j} / 2\right)=y_{j}$, a distribuição fica sendo

$$
\begin{aligned}
& P_{\mathcal{S O}(2 N+1)}\left(y_{1}, \ldots, y_{N}\right) \\
& \propto \prod_{j=1}^{N} y_{j}^{-1 / 2}\left(1-y_{j}\right)^{1 / 2} \prod_{1 \leq j<k \leq N}\left(y_{k}-y_{j}\right)^{2},
\end{aligned}
$$

$\operatorname{com} 0 \leq y_{j} \leq 1$.

Feito isso, é possível calcular o valor médio de várias funções simétricas dos autovalores (relacionadas ao polinômio característico da matriz, por exemplo) usando a famosa integral de Selberg:

$$
\begin{gathered}
S(a, b, \gamma)=\int_{(0,1)^{N}} d y \prod_{j=1}^{N} y_{j}^{a-1}\left(1-y_{j}\right)^{b-1} \\
\prod_{1 \leq j<k \leq N}\left|y_{k}-y_{j}\right|^{2 \gamma},
\end{gathered}
$$

cujo resultado é

$$
S(a, b, \gamma)=\prod_{j=0}^{N-1} \frac{\Gamma(a+j \gamma) \Gamma(b+j \gamma) \Gamma(1+(j+1) \gamma)}{\Gamma(a+b+(n+j-1) \gamma) \Gamma(1+\gamma)}
$$

Note que, fazendo $x_{j}=2 y_{j}-1$, podemos expressar a integral em termos das variáveis $x$.

Essa integral é uma generalização para muitas variáveis da clássica integral de Euler,

$$
\int_{0}^{1} d y y^{a-1}(1-y)^{b-1}=\frac{\Gamma(a) \Gamma(b)}{\Gamma(a+b)} .
$$

É interessante observar que, assim como os clássicos polinômios de Jacobi $P_{n}^{a, b}(y)$ são ortogonais em relação ao integrando de Euler

$$
\int_{0}^{1} d y y^{a-1}(1-y)^{b-1} P_{n}^{a, b}(y) P_{m}^{a, b}(y) \propto \delta_{n m},
$$


é possível definir polinômios em várias variáveis que são ortogonais em relação ao integrando de Selberg (ver [17]). Nos casos particulares das equações (34) e (35), em que $(a, b)=\left(\frac{1}{2}, \frac{1}{2}\right)$ e $\left(\frac{1}{2}, \frac{3}{2}\right)$, respectivamente, esses polinômios são os caracteres do grupo, que mencionamos a seguir.

\subsection{Caracteres}

Outra forma de abordar os autovalores é escrever sua distribuição em termos dos números complexos $z_{k}=e^{i \theta_{k}}$ e $\bar{z}_{k}=e^{-i \theta_{k}}$. Nesse caso, temos

$$
\begin{aligned}
& P_{\mathcal{S O}(2 N)}\left(z_{1}, \ldots, z_{N}, \bar{z}_{1}, \ldots, \bar{z}_{N}\right) \\
& \propto \prod_{1 \leq j<k \leq N}\left|z_{k}-z_{j}\right|^{2}\left|1-z_{k} z_{j}\right|^{2}
\end{aligned}
$$

e

$$
\begin{aligned}
& P_{\mathcal{S O}(2 N+1)}\left(z_{1}, \ldots, z_{N}, \bar{z}_{1}, \ldots, \bar{z}_{N}\right) \\
& \quad \propto \prod_{j=1}^{N}\left|1-z_{j}\right|^{2} \prod_{1 \leq j<k \leq N}\left|z_{k}-z_{j}\right|^{2}\left|1-z_{k} z_{j}\right|^{2}
\end{aligned}
$$

Essas distribuições admitem uma família de polinômios ortogonais, que generalizam a relação $\oint z^{n} \bar{z}^{m} \propto \delta_{n m}$, na qual a integral é realizada sobre o círculo unitário. Além de terem várias variáveis, esses polinômios também têm vários índices inteiros, tradicionalmente denotados por $\lambda=\left(\lambda_{1}, \lambda_{2}, \ldots\right)$ com $\lambda_{i} \geq \lambda_{i+1}$. Eles são dados por 18

$$
\begin{aligned}
o_{\lambda}^{(2 N)}\left(z_{1}, \ldots, z_{N}, \bar{z}_{1}, \ldots, \bar{z}_{N}\right) \\
=\frac{\operatorname{det}\left(z_{i}^{N+\lambda_{j}-j}+\bar{z}_{i}^{N+\lambda_{j}-j}\right)}{\operatorname{det}\left(z_{i}^{N-j}+\bar{z}_{i}^{N-j}\right)}
\end{aligned}
$$

em dimensão par e

$$
\begin{aligned}
o_{\lambda}^{(2 N+1)}\left(z_{1}, \ldots, z_{N}, \bar{z}_{1}, \ldots, \bar{z}_{N}\right) \\
=\frac{\operatorname{det}\left(z_{i}^{N+\lambda_{j}-j+1 / 2}-\bar{z}_{i}^{N+\lambda_{j}-j+1 / 2}\right)}{\operatorname{det}\left(z_{i}^{N-j+1 / 2}-\bar{z}_{i}^{N-j+1 / 2}\right)}
\end{aligned}
$$

em dimensão ímpar. Os determinantes são polinômios anti-simétricos, enquanto que a razão entre eles produz um polinômio simétrico.

Vamos demonstrar essa ortogonalidade $\oint o_{\lambda}^{(2 N)} o_{\mu}^{(2 N)} \propto$ $\delta_{\lambda \mu}$, nos restringindo à dimensão par por simplicidade. Primeiro, usamos o fato de que

$$
\begin{aligned}
\operatorname{det}\left(z_{i}^{N-j}+\bar{z}_{i}^{N-j}\right) & \propto \prod_{1 \leq j<k \leq N}\left(z_{k}-z_{j}\right)\left(1-\bar{z}_{k} \bar{z}_{j}\right) \\
& \propto \prod_{1 \leq j<k \leq N}\left(\bar{z}_{k}-\bar{z}_{j}\right)\left(1-z_{k} z_{j}\right) .
\end{aligned}
$$

Como temos duas funções na integral, esse denominador aparece duas vezes e cancela com os termos presentes na medida 40. Ficamos então com

$$
\begin{array}{r}
\oint o_{\lambda}^{(2 N)} o_{\mu}^{(2 N)} \propto \oint \operatorname{det}\left(z_{i}^{N+\lambda_{j}-j}+\bar{z}_{i}^{N+\lambda_{j}-j}\right) \\
\operatorname{det}\left(z_{i}^{N+\mu_{j}-j}+\bar{z}_{i}^{N+\mu_{j}-j}\right) .
\end{array}
$$

Recorremos agora a um teorema conhecido como identidade de Andreief, que relaciona uma integral múltipla, sobre $N$ variáveis, de dois determinantes ao determinante de uma matriz $N \times N$, cujos elementos são integrais simples:

$$
\int \operatorname{det}\left(f_{i}\left(z_{j}\right)\right) \operatorname{det}\left(g_{j}\left(z_{i}\right)\right)=N ! \operatorname{det}\left(\int f_{i}(z) g_{j}(z)\right) .
$$

Aplicada ao nosso caso, essa identidade fornece

$$
\begin{aligned}
& \oint o_{\lambda}^{(2 N)} o_{\mu}^{(2 N)} \\
& \propto \operatorname{det}\left(\oint\left(z^{N+\lambda_{i}-i}+\bar{z}^{N+\lambda_{i}-i}\right)\left(z^{N+\mu_{j}-j}+\bar{z}^{N+\mu_{j}-j}\right)\right)
\end{aligned}
$$

$$
\propto \operatorname{det}\left(\oint\left(z^{N+\lambda_{i}-i} \bar{z}^{N+\mu_{j}-j}+\bar{z}^{N+\lambda_{i}-i} z^{N+\mu_{j}-j}\right)\right)
$$

$\propto \operatorname{det}\left(\delta_{\lambda_{i}-i, \mu_{j}-j}\right) \propto \delta_{\lambda \mu}$.

Para o leitor que quiser verificar a ortogonalidade em coordenadas reais, oferecemos um exemplo em $N=2$. Nesse caso,

$$
o_{(1,1)}^{(4)}=1+2 \cos \left(\theta_{1}\right) \cos \left(\theta_{2}\right)
$$

$\mathrm{e}$

$$
o_{(2,0)}^{(4)}=2 o_{(1,1)}^{(4)}+2 \cos \left(2 \theta_{1}\right)+2 \cos \left(2 \theta_{2}\right)-1
$$

enquanto que $P_{\mathcal{S O}(4)} \propto\left(\cos \left(\theta_{2}\right)-\cos \left(\theta_{1}\right)\right)^{2}$.

Em termos mais gerais da teoria de grupos, as funções $o_{\lambda}^{(N)}$ são ortogonais porque correspondem aos caracteres das representações irredutíveis do grupo ortogonal $\mathcal{O}(N)$. Esses polinômios formam uma base para o espaço dos polinômios que são simétricos nos autovalores. Assim, a integral de qualquer função desse tipo pode ser calculada expandindo-a na base ortogonal. Para uma exposição dessa teoria, recomendamos [19].

\section{Conclusão}

Apresentamos neste artigo uma discussão introdutória sobre rotações aleatórias, acessível a estudantes de graduação em física. Fizemos a distinção entre rotações e reflexões e introduzimos a medida de Haar sobre o grupo ortogonal, o que permite calcular diversas quantidades de interesse estatístico. O tema mais geral das matrizes 
aleatórias encontra inúmeras aplicações e integrais sobre o grupo ortogonal foram recentemente usadas em trabalhos do nosso grupo de pesquisa, por exemplo em [20 23]. Esperamos que esta apresentação possa ajudar a disseminar o interesse por esse tema.

M. Novaes foi financiado pelos processos 306765/20187 e 400906/2016-3 do CNPq. L.H. Oliveira tem bolsa de doutorado da Capes. Agradecemos a um parecerista anônimo por suas excelentes contribuições à clareza do artigo.

\section{Referências}

[1] M.L. Mehta, Random Matrices (Elsevier, Amsterdam, 2004).

[2] R.J. Muirhead, Aspects of Multivariate Statistical Theory (John Wiley \& Sons, Hoboken, 2009).

[3] K. Binder, Glassy Materials and Disordered Solids (World Scientific, Singapore, 2011).

[4] F. Haake, Quantum Signatures of Chaos (Springer, Cham, 2019), 4th ed.

[5] R. Brannon, Rotation, Reflection and Frame Changes (Institute of Physics, Bristol, 2018).

[6] J.B. Kuipers, Quaternions and Rotation Sequences (Princeton University Press, Princeton, 2002).

[7] W.J. Thompson, Angular Momentum (Wiley, New York, 1994).

[8] J. Gallier, Geometric Methods and Applications (Springer, New York, 2011).

[9] J. Stillwell, Naive Lie Theory (Springer, New York, 2008).

[10] P. Diaconis e P.J. Forrester, arXiv:1410.6535v1 (2016).

[11] Haar measure on $\mathcal{S O}(n)$. Disponível em: https://math. stackexchange.com/questions/1364495/haar-measureon-son

[12] F. Mezzadri, Notices Am. Math. Soc. 54, 592 (2007).

[13] B.A. Khoruzhenko, H.-J. Sommers e K. Życzkowski, Phys. Rev. E. 82, 040106(R) (2010).

[14] B. Collins e S. Matsumoto, J. Math. Phys. 50, 113516 (2009).

[15] G. Wang, Y. Wei e S. Qiao, Generalized Inverses (Springer, Singapore, 2018).

[16] H. Weyl, The Classical Groups: their Invariants and Representations (Princeton University Press, Princeton, 1939).

[17] P.J. Forrester e S.O. Warnaar, Am. Math. Soc. 45, 489 (2008).

[18] E.S. Meckes, The Random Matrix Theory of the Classical Compact Groups (Cambridge University Press, Cambridge, 2019).

[19] R. Goodman e N.R. Wallach, Symmetry, Representations, and Invariants (Springer, New York, 2009).

[20] M. Novaes, Ann. Phys. 361, 51 (2015).

[21] M. Novaes, J. Math. Phys. 57, 122105 (2015).

[22] P.H.S. Palheta, M.R. Barbosa e M. Novaes, arXiv: 2004.09266 (2020).

[23] L.H. Oliveira e M. Novaes, arXiv:2002.08112 (2020). 